\title{
Traduire
}

Revue française de la traduction

234 | 2016

La traduction, un sport de haut niveau

\section{Une correspondante sportive dans l'abîme du décalage langagier}

Hiroko Nakao

\section{(2) OpenEdition}

Journals

Édition électronique

URL : http://journals.openedition.org/traduire/799

DOI : $10.4000 /$ traduire.799

ISSN : 2272-9992

Éditeur

Société française des traducteurs

Édition imprimée

Date de publication : 15 juin 2016

Pagination : 43-46

ISSN : 0395-773X

\section{Référence électronique}

Hiroko Nakao, « Une correspondante sportive dans l'abîme du décalage langagier », Traduire [En ligne], 234 | 2016, mis en ligne le 15 juin 2018, consulté le 01 mai 2019. URL : http://journals.openedition.org/ traduire/799 ; DOI : 10.4000/traduire.799 


\section{Une correspondante sportive dans l'abîme du décalage langagier}

\section{Hiroko Nakao}

Depuis 2006, l'année de mes débuts professionnels en tant que traductrice-interprète, mes travaux se sont progressivement concentrés sur la traduction dans le domaine des sports, notamment celui du football : interprétation consécutive lors d'une conférence de presse du Grenoble Foot 38, création et mise à jour de contenus sur le serveur d'un site officiel spécialement conçu pour la Coupe du Monde, préparation d'informations destinées aux commentateurs des matchs de Ligue 1 diffusés au Japon, rédaction d'articles de fond pour le compte de la revue hebdomadaire Footballista... Toutes ces expériences se sont avérées uniques, très intéressantes, et littéralement " sportives ". Sportives, car les délais étaient souvent extrêmement courts, d'une part en raison du décalage horaire, et d'autre part parce que la concurrence avec les autres médias nécessitait de fournir des informations les plus récentes possible. Sportives encore, car il en a fallu de l'endurance pour suivre les équipes avec tous les moyens de transport imaginables et courir derrière un joueur ou un entraîneur qui refusait parfois de répondre à une interview.

La plus représentative de mes expériences de traduction dans le monde du sport remonte à la période 2006-2011, époque où je travaillais en tant que correspondante pour le quotidien japonais Sports Nippon. J'ai ainsi vécu et suivi de près la reprise du club de foot grenoblois par l'entité japonaise Index, son projet de construction et les épisodes judiciaires des permis de construire du nouveau stade, l'ascension du club vers la lumière glorieuse de la première division, sa décadence progressive puis... la faillite financière et la débâcle sportive qui ont clos, pour l'instant, l'histoire professionnelle du club.

\section{La journée type d'un correspondant}

Concrètement, le travail de correspondant sportif est varié et dépasse largement le cadre habituel du traducteur ou de l'interprète, même si le point fort lors de mon recrutement fut ma maîtrise de la langue française. Ma mission consistait à suivre les joueurs japonais des clubs 
de football français, notamment au Grenoble Foot 38, et ceux qui étaient de passage en France pour des matchs internationaux ou européens.

La journée débutait par l'achat de plusieurs quotidiens dès l'ouverture de la maison de la presse à $7 \mathrm{~h}$ 30. De retour chez moi, je me plongeais dans les rubriques dédiées au football et rédigeais un résumé des articles les plus intéressants (ou en effectuais une traduction intégrale sur demande). De plus, trois fois par semaine au moins (veille de match du week-end, lendemain de match et milieu de semaine), j'allais sur le terrain d'entraînement pour évaluer la session et faire mon rapport à la rédaction par téléphone. La visite la plus importante était celle de la veille de match, quand il fallait interviewer l'entraîneur pour connaître la composition de l'équipe titulaire et obtenir quelques commentaires de la part des joueurs japonais. Tous les éléments réunis étaient immédiatement transmis par téléphone au Japon. Quant au rapport écrit, il devait suivre au plus vite par courriel.

$\mathrm{Si}$, à ce moment-là, on apprenait qu'un joueur japonais était titularisé ou faisait au moins partie du groupe pour un match extérieur, il fallait que je sois prête à partir en moins de $24 \mathrm{~h}$ pour y assister. L'organisation du voyage devait se faire en un clin d'œil : transport, hôtel, localisation du stade, demande d'accréditation, préparation de la valise... J'ai ainsi pu voyager partout en France, passant par l'imposant stade lyonnais et sa folle soirée du championnat de France, le stade bordelais pour un match de la Ligue des Champions, ou encore celui de Porto Vecchio en CFA2 (amateur) dans le sud de l'Île de beauté. Le côté dynamique et excitant du métier ne fait pourtant pas oublier l'autre facette, omniprésente elle aussi : la gestion des relations humaines et des interactions langagières, qui peut se révéler assez compliquée.

\section{Le choix fâcheux d'un mot}

Voici une petite anecdote pour mieux illustrer mon propos. Quelques jours après l'officialisation du transfert d'un joueur japonais à Grenoble, ce dernier avait immédiatement intégré le groupe pour un match à domicile. Mais étant remplaçant, il était resté cloué sur le banc durant tout le match. Très consciencieuse, j'avais rapporté à la rédaction qu'» il n'avait pas eu la chance de jouer pour son premier match en France, bien qu'ayant intégré le groupe ". L'article du quotidien sorti le lendemain mentionnait : " $\|$ a terminé son premier match comme réserve 》(控え).

Le jour même, mon téléphone sonna. Le directeur général japonais du Grenoble Foot en personne, furieux, m'accusait d'avoir usé d'un terme trop négatif pour traduire " remplaçant ". II exigeait que j'emploie l'expression ベンチスタート (littéralement : joueur débutant sur le banc). J'ai appelé au Japon pour exposer cette réclamation à la rédaction, car la menace était suffisamment claire : le directeur général m'avait fait comprendre que si nous continuions à utiliser ce terme, le quotidien pourrait se voir interdire d'approcher le joueur. 
La rédaction, qui tombait des nues, me donna sa version des faits : le mot 控え, en deux caractères seulement, avait été préféré du fait d'une réduction de longueur de l'article en dernière minute. En effet, au regard de son temps de jeu nul, il ne semblait pas utile de s'étendre sur ce joueur. Le terme ベンチスタート, comportant sept caractères, était ainsi devenu trop long. Pour que la rédaction accepte que le terme long soit employé, il fallait que le joueur ait déjà joué, voire marqué des buts ! Entre la direction du club très attentive à son image (voire nerveuse) et les journalistes qui rédigeaient un article sur la base de mes informations et n'imaginaient pas une seconde qu'un tel conflit d'intérêts puisse naître du choix d'un mot, j'ai dû ajouter pendant longtemps, à la fin de chaque rapport, la note suivante : " Merci de ne pas utiliser le terme 控え invalidé par le club pour sa connotation négative".

\section{Le choc thermique entre le Japon et la France}

En ce qui concerne la traduction des articles de presse, la difficulté consistait à faire comprendre aux lecteurs les jeux de mots, les " non-dits " que l'on peut lire entre les lignes, l'ironie des tournures françaises... bref, traduire l'intraduisible.

Prenons un article évoquant le transfert d'un deuxième joueur japonais, un lycéen de 18 ans à l'époque, qui n'avait pas encore fini ses études secondaires. Commençant sa carrière comme professionnel en France sur les chapeaux de roue, ce joueur, surnommé au Japon le «Thierry Henri japonais ", était l'espoir du pays. Toutefois, les Français le considéraient comme un jeune en cours de formation, ce qui était d'ailleurs tout à fait vrai car il n'avait aucun titre, aucune expérience et n'était pas encore au maximum de ses possibilités physiques. Les Français, en toute logique, suivaient la conférence de presse électrisée autour de ce jeune espoir d'un œil plutôt ironique : ils parlaient d'un joueur "à la sauce japonaise ", "baignant " dans le giron confortable des dirigeants nippons. Afin d'expliquer cette attitude hautaine, voire goguenarde, j'ai dû ajouter maintes notes à la traduction intégrale de cet article à l'attention de la rédaction. Pourtant, comme il fallait s'y attendre, ces commentaires négatifs de la part des Français - et mes notes explicatives - passèrent allègrement à la trappe, me laissant l'impression amère d'un fâcheux décalage entre la réalité et le fait raconté.

Ce décalage se faisait sentir au quotidien. La rédaction préférait publier du sensationnel pour capter l'attention, comme on l'observe souvent dans le monde de la presse. Mais aux yeux de quelques lecteurs internes du club, certains passages anodins ou mots employés sans mauvaise intention n'étaient que manipulation. Un phénomène d'exagération continua de creuser l'abîme entre le club et les correspondants qui recevaient systématiquement des réclamations par téléphone, car pour le club nous étions les représentants directs du journal pour lequel nous traduisions. Ce choc thermique fut également palpable dans les articles du journal régional 
français. Les expressions qui reflétaient la vision d'un mur infranchissable entre les deux cultures étaient récurrentes : " distance ", "incompréhension ", " difficulté ", " pays lointain ", " impossibilité de communiquer ", " absence d'interlocuteur ", etc.

Si l'aventure du Grenoble Foot 38 et de la société Index a pris fin, c'est bien entendu essentiellement pour des raisons financières. Cependant, le décalage entre les attentes des uns et la réalité des autres, sur fond de procès d'intention culturel ou de fatalisme linguistique, a clairement joué un rôle de sape. Je me suis amèrement demandé si je n'avais pas participé malgré moi à creuser ce gouffre. Malheureusement, c'est un phénomène classique que l'on peut observer dans le monde de la presse. Pour ma part, je garde un sentiment d'inaccompli qui m'a poussée à écrire des articles de fond sur le football avec mes propres mots. Je peux désormais mettre mon expérience en relation avec des phénomènes de société, l'actualité, les affaires juridiques... Mais tout cela est une autre histoire.

info@bureau-traduction.eu

Hiroko Nakao est interprète et traductrice indépendante japonais-français dans les domaines juridique et sportif. Elle est également expert près la Cour d'appel de Grenoble depuis 2007.

\section{DOPAGE}

La fédération continue de fermer les yeux sur certains produits énergisants

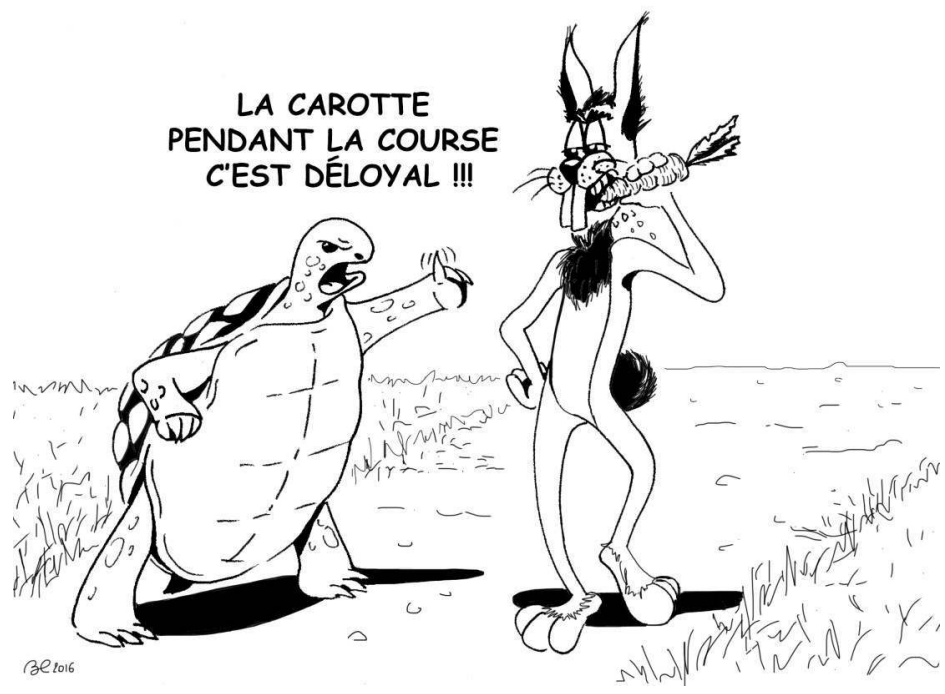

